\title{
Gastrointestinal Leiomyosarcoma with Disease Progression to the Central Nervous System: Case Report
}

Joao Gustavo Rocha Peixoto dos Santos ${ }^{*}$, luri Santana Neville, Luiz Guilherme Cernaglia Aureliano Lima, Guilherme Alves Lepski and Manoel Jacobsen Teixeira

Department of Neurosurgery, University of Sao Paulo School of Medicine, Sao Paulo, Brazil

"Corresponding author: Joao Gustavo Rocha Peixoto dos Santos, Department of Neurosurgery, University of Sao Paulo School of Medicine, Sao Paulo, Brazil, Tel: 55 11 3091-3116; E-mail: joao_gustavo@icloud.com

Rec date: Aug 14, 2016; Acc date: Aug 18, 2016; Pub date: Aug 20, 2016

Copyright: ( 2016 Santos JGRP, et al. This is an open-access article distributed under the terms of the Creative Commons Attribution License, which permits unrestricted use, distribution, and reproduction in any medium, provided the original author and source are credited.

\begin{abstract}
Introduction: Leiomyosarcomas (LMS) are rare malignant neoplasms with smooth muscle differentiation, which occur more commonly in the uterus, digestive tract or retroperitoneum. Before 2000, the mesenchymal gastric tumors were divided in leiomyomas, leiomyoblastomas and leiomyosarcomas. After discovery of the role of KIT oncogene, a distinct entity in the mesenchymal gastrointestinal tumors line has surfaced: GIST (Gastrointestinal Stomal Tumors). The true gastric leiomyosarcoma has become a rare entity. The presentation with brain metastasis had only been reported in the pre-GIST era.
\end{abstract}

Case report: A female, 57 years old, admitted in October 2013, for investigation of abdominal pain, finding a lesion in the left illiac fossa continuing with the terminal ileum. She was submitted to the resection of the lesion with histopathological result showing leiomyosarcoma (6 mitotic figures/10 high power field; IHQ: vimentin positive, S-100 negative, desmin positive). Started chemotherapy. However, presented progression of the disease for subcutaneous tissue, liver (submitted to radiotherapy) and nervous system (only one lesion in the posterior fossa). The patient was submitted in March 7th 2016 to suboccipital craniotomy and en-block resection of the lesion. The microscopic examination revealed a fasicular spindle cell malignant neoplasm, with necrotic areas, with intense and diffuse desmin and smooth muscle actin-positivity in immunohistochemical study, which confirmed the leiomyosarcoma metastatic lesion hypothesis. The patient evolved well during the postoperative period, it was peformed the postoperative MRI, that showed a satisfactory resection. She remained hospitalized until the fourth postoperative day, with no complications, and was then discharged for outpatient follow-up.

Conclusion: We have described a case of gastric leiomyosarcoma, emphasizing that even in front of the rarity of leiomyosarcoma itself as a primary lesion (especially after the discovery of GIST as an independent mesenchymal line tumor), this lesion may progress and present central nervous system metastasis, a fact not before reported in literature.

Keywords: Leiomyosarcoma; Metastasis; Central nervous system; Posterior fossa; Gastrointestinal; Pathology; Gastointestinal stromal tumor

\section{Introduction}

Leiomyosarcomas (LMS) are rare malignant neoplasms with smooth muscle differentiation, which occur more commonly in the uterus, digestive tract or retroperitoneum [1]. It is an entity with reserved prognosis due to the high probability of metastatic presentation, the lungs, peritoneal cavity and liver being the most strickensites, respectively [1].

Before 2000, the mesenchymal gastric tumors were divided in leiomyomas, leiomyoblastomas and leiomyosarcomas. After discovery of the role of KIT oncogene, a distinct entity in the mesenchymal gastrointestinal tumors line has surfaced: GIST (Gastrointestinal Stomal Tumors) [1,2]. GISTs are characterized by the expression or function gain with mutation of KIT protein or the presence of the codifying gene for the platelet derived growth factor receptor alpha (PDGFRA) [3]. Thus, the true gastric leiomyosarcoma has become a rare entity. The presentation with brain metastasis had only been reported in the pre-GIST era [4-7].

The true gastric leiomyosarcomas constitute $1-3 \%$ of all the gastric tumors, representing less than $1 \%$ of all the primary gastric neoplasias. As is expected of a rare disease, diagnostic related descriptions, therapeutic options and prognostic determinants are few and generally with inconsistent and limited conclusions due to the restricted number of reported cases [8]. There is also in the literature the description of the primary central nervous system leiomyosarcoma, which origin is believed to be related to blood vessel smooth muscle [9].

The goal of the presented report is to describe a case of a patient with the diagnosis of gastrointestinal leiomyosarcoma with central nervous system metastasis, in follow up at Instituto do Cancer do Estado de Sao Paulo Octavio Frias de Oliveira (ICESP).

\section{Case Report}

It is a report of the patient D.R.N.K., female, 57 years old, admitted in October 2013, for investigation of abdominal pain associated with nausea and vomiting. It was performed a computerized tomography of 
Citation: Santos JGRP, Lima LGCA, Neville IS, Lepski GA, Teixeira MJ (2016) Gastrointestinal Leiomyosarcoma with Disease Progression to the Central Nervous System: Case Report. J Neurol Disord 4: 291. doi:10.4172/2329-6895.1000291

Page 2 of 3

abdomen which identified a lesion in the left iliac fossa continuing with the terminal ileum.

The patient was thus submitted to resection of the lesion in the same hospitalization period with histopathological result showing leiomyosarcoma (6 mitotic figures/10 high power field; IHQ: vimentin positive, S-100 negative, desmin positive, actin positive, CD 34 positive, CD 117 (ckit) negative; DOG-1: focally positive). In December of that year, it was initiated chemotherapy with Imatinib mesylate (maintained until March/2014) and another tomography for staging was performed, showing a secondary hepatic lesion and 3 subcutaneous lesions (occipital region, cervical and dorsal).

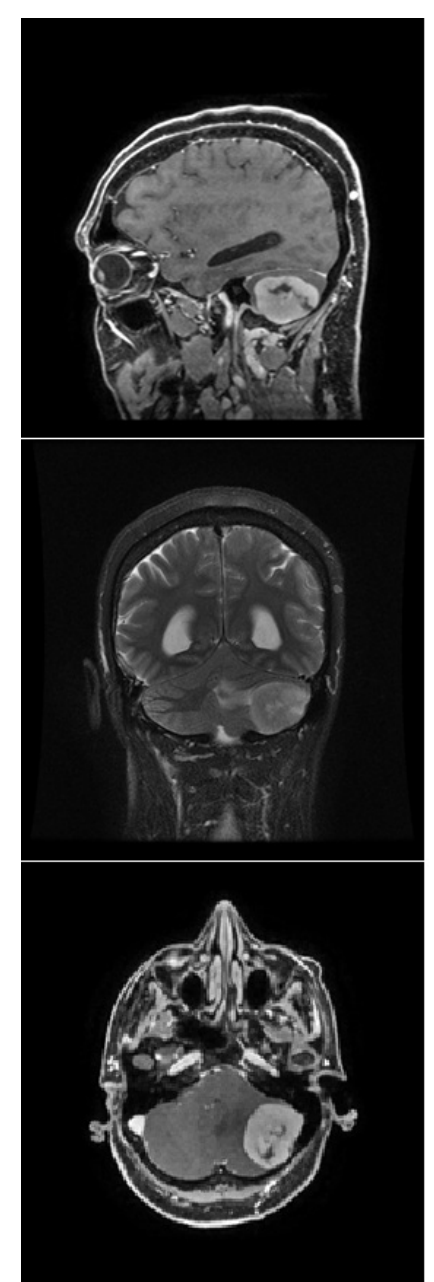

Figure 1: Pre-operative MRI.

The subcutaneous nodules presented a hardened and fibroelastic consistency. The largest in the dorsal region measured about $3.5 \times 3.5$ $\mathrm{cm}$ and it was easily identifiable and palpable. It was opted for hypofractionated radiotherapy ( 05 x 1000c Gy until 04/08/2014) of the hepatic lesion and biopsy of one of the subcutaneous lesions. The histopathological result of the new biopsy reveled a fusiform cell neoplasia, compatible with leiomyosarcoma metastasis (Desmin positive, CD34 focal positive, C-KIT, RE, DOG1, S100 negative).

In February of 2016, patient presented difficulty reaching objects with the left hand and vertigo. Investigated with encephalon MRI which showed disease progression for the central nervous system with metastasis in the left posterior fossa (Figure 1).

The patient was submitted in March $7^{\text {th }} 2016$ to sub occipital craniotomy and en-block resection of the lesion. During surgery it was visualized a base lesion in tentorial dura mater (meningotelial), hardened and respecting the arachnoid plan with the cerebellum. It was performed en-block resection and forwarded the lesion for histopathological analysis.

The microscopic examination revealed a fascicular spindle cell malignant neoplasm, with necrotic areas, with intense and diffuse desmin and smooth muscle actin-positivity in immunohistochemical study, which confirmed the leiomyosarcoma metastatic lesion hypothesis (Figures 2-6).

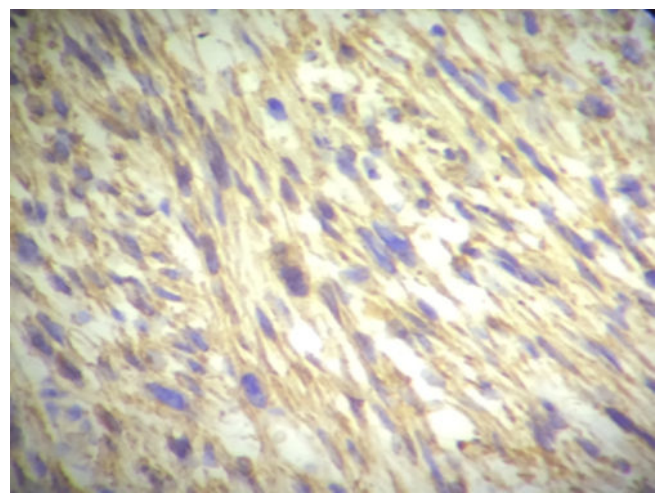

Figure 2: Immunohistochemistry (Actin 400x).

Interestingly, with no clinical date, this diagnosis would be harder to conclude, once this histologic feature could be found in meningeal sarcomas, malignant meningiomas and gliosarcomas, which mean a different therapeutically approach.

The patient evolved well during the postoperative period, it was performed the postoperative MRI, that showed a satisfactory resection. She remained hospitalized until the fourth postoperative day, with no complications, and was then discharged for outpatient follow-up.

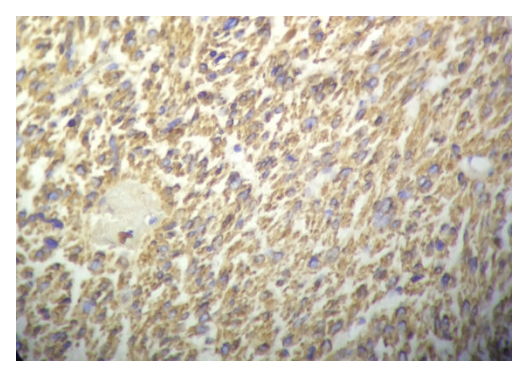

Figure 3: Immunohistochemistry (Desmin 400x). 


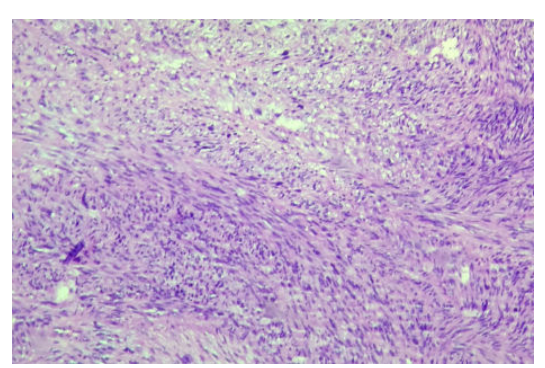

Figure 4: LMS 200x - Fasciculas and spindles cells.

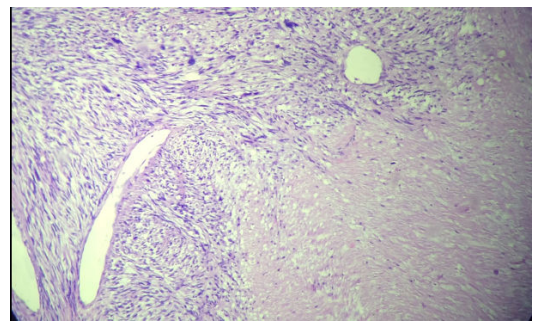

Figure 5: LMS 100x Necrotic areas.

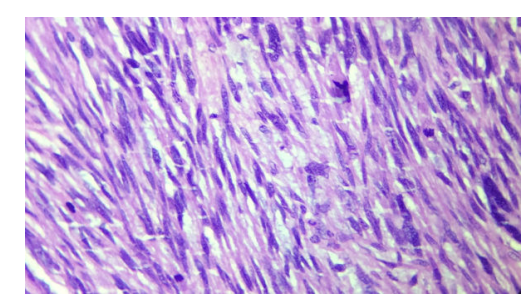

Figure 6: LMS 400x Cytologic features.

\section{Discussion}

The Leiomysarcoma is a part of the malignant neoplasia originated more commonly from the smooth muscle of the uterus, gastrointestinal tract or blood vessels [7]. It has a poor prognosis with survival rate in five years varying from 0 to $68 \%$ in several series in literature [10]. One of the largest determinants of this prognosis is the high rate of metastasis, which usually occur through hematogenous pathway (the lung being the main target) and through lymphatic pathway [7]. The patient in the reported case presented secondary lesions in liver (treated with fractionated radiotherapy) and subcutaneous skin tissue (occipital and dorsal regions), with no evidence of disease in other sites.

If the leiomyosarcoma entity was already considered rare, after the modern concept of differential with GIST entity, it has become extremely rare [2]. It is reported that leiomyosarcoma frequency is $1 / 15$ of the GISTs [2]. The present case shows two different metastatic histopathological results, besides the one obtained in the primary site, demonstrating itself a different entity from GIST.
The image characteristics are variable and unspecific, however they generally present with high signal and homogeneous contrast capitation [7]. These characteristics are not specific and are similar to a meningioma, for example [7]. In our case the lesion showed isointense in T1, mildly hiper intense in T2 and FLAIR with preserved arachnoid plan (extra-axial lesion) with a homogeneous capitation of gadolinium and dural tail due to the relation and meningothelial origin, with base dura mater of the posterior fossa. These characteristics are in fact not specific, found also in other cerebral metastatic lesions.

There is description of several other intracranial leiomyosarcoma cases [4-7], however none of them after the differentiation of leiomyosarcoma with GIST entity. It is not possible, therefore, to conclude which of them where in fact leiomyosarcomas, however it is likely that some of them were. In the presented patient, in turn, the histopathology was tested and differentiated with help of immunohistochemistry (absence of cKIT and PDGRFA). It is, therefore the first case report with confirmed histopathological diagnosis after the description of GIST as a distinct histopathological entity from the leiomyosarcomas.

\section{Conclusion}

We have described a case of gastric leiomyosarcoma, emphasizing that even in front of the rarity of leiomyosarcoma itself as a primary lesion (especially after the discovery of GIST as an independent mesenchymal line tumor), this lesion may progress and present central nervous system metastasis, a fact not before reported in literature (in the post-GIST era).

\section{References}

1. Pereira FOC, Pereira DC, Aguiar RC, Lombardi IAS, Zanini MA (2011) Leiomyosarcoma metastatic to the skull and spine: A case report. Revista Brasileira de Cancerologia 57: 63-66.

2. Yamamoto H, Handa M, Tobo T, Setsu N, Fujita K, et al. (2013) Clinicopathological features of primary leiomyosarcoma of the gastrointestinal tract following recognition of gastrointestinal stromal tumours. Histopathology 63:194-207.

3. Lasota J, Miettinen M (2006) KIT and PDGFRA mutations in gastrointestinal stromal tumors (GISTs). Semin Diagn Pathol 23: 91-102.

4. Adachi M, Wellman KF, Garcia R (1969) Metastatic leiomyosarcoma in brain and heart. J Pathol 98: 294-296.

5. Gercovich FG, Luna MA, Gottlieb JA (1975) Increased incidence of cerebral metastasis in sarcoma patients with prolonged survival from chemotherapy (1975) Report of cases of leiomyosarcoma and chondrosarcoma. Cancer 36: 1843-1851.

6. Sato S, Kambra H, Hotta J (1972) A case of leiomyosarcoma with fatal intracerebral metastasis. Seikagaku 23: 381-384.

7. Haykal H, Wang AM, Zamani A (1987) Leiomyosarcoma metastatic to the brain: CT Features and Review. AJNR 8: 911-912.

8. Burchett K, Carungi R (1999) Gastric leiomyosarcoma presenting as a sentinel hemorrhage. JAOA 10: 533-536.

9. Ferreira A, Garcia V, Costa MV, Braga FM (1996) Leiomiossarcoma cerebral. J Bras Neurocirurg 7: 50-52.

10. Prussia PR, Clarke HA, Mansoor G, Garriques S, Maheswaran B (1992) Uterine leiomyosarcoma with intracerebral metastasis: a case report. J Natl Med Assoc 84: 368-370. 